\title{
The Temporal Dimension of Copresence in Medical Practice: The Case of Telestroke
}

\begin{abstract}
This paper examines how co-presence is enacted in technology-mediated medical practices, particularly under time pressure. Extant literature highlights time (e.g. immediacy and duration of interactions) as a critical condition for copresence, but there has been little attention to the variation of copresence over time. In this paper, we investigate this variation through an ethnographic study in three emergency departments that are linked via a telemedicine system called Telestroke, which is used to diagnose and treat stroke patients at a distance. We draw on the sensemaking literature to uncover how copresence is enacted across different phases of technology-mediated medical practice. Our findings reveal four mechanisms that shape the variation of copresence across time, namely extracting cues, retrospection, perspective-taking, and selective attention.
\end{abstract}

\section{Keywords:}

Copresence, Sensemaking, Ethnography, Telemedicine, Time-Pressure. 


\section{The Temporal Dimension of Copresence in Medical Practice: The Case of Telestroke Introduction}

The presence of clinicians is often essential throughout the different phases of healthcare delivery, including diagnosis, treatment decision and administration, and patient follow-up. Medical practice commonly requires close interaction and direct contact between healthcare professionals and patients. The projection of a sense of "care" and attention to the wellbeing of the patient is critical (Henderson, 2006). The socio-cognitive process that shapes this requirement has been widely referred to as copresence, which is a concept that reflects the general perception of being present in a mediated interaction (Short, Williams, \& Christie, 1976).

For the patients, copresence contributes to decreasing the level of anxiety (Liu et al., 2017). For the clinicians, copresence creates a sense of closeness to the patient, which improves the quality of care (Paul et al., 2017). In non-healthcare settings, higher levels of copresence have been shown to lead to enhanced trust (Ou, Pavlou, \& Davison, 2014; Srivastava \& Chandra, 2018) and higher group performance (Altschuller \& Benbunan-Fich, 2010; Yoo \& Alavi, 2001).

Attaining these benefits of copresence requires an understanding of the mechanisms that generate it and sustain it over time. However, extant literature has largely investigated copresence at single points in time and did not consider its variation over time. Temporality has been an important element in the theoretical developments of the notion of copresence (Ma \& Agarwal, 2007). For example, the duration of mediated interactions and the immediacy of response have been studied as critical factors in attaining copresence (Harrison, 2018). Longer interactions facilitate the flow of information and reduce uncertainty and are, therefore, more conducive to copresence (Srivastava \& Chandra, 2018). However, changes in the need for 
copresence over time and in its actual enactment in practice have received little theoretical attention to date. Thus, in this paper, we ask the following question: what mechanisms influence the variation of copresence in technology-mediated environments?

To address this question, we draw on an ethnographic study in three emergency departments that collaborate at a distance to treat stroke patients by using a telemedicine system called "Telestroke." This setting offers a revealing case for our research question because the treatment of stroke involves multiple phases. Time is a critical factor for the survival of stroke patients. The time constraints hence make the achievement of copresence in and of itself challenging. We identify four mechanisms that actors use as an approach to vary their level of copresence under time pressure, namely extracting cues, retrospection, perspective-taking, and selective attention.

\section{Literature Review}

Copresence is defined as "the degree of salience of the other person in the interaction and the consequent salience of the interpersonal relationship" (Short et al., 1976; Yoo \& Alavi, 2001). In this section, we first provide an overview of the importance of copresence in the context of medical services. Second, we present a review of the literature on the conditions necessary for achieving copresence in distributed work. Finally, we discuss copresence through the lens of sensemaking theory.

\section{Copresence in Distributed Work}

Scholars have been interested in copresence ever since Goffman (1982) coined the term to highlight it as an essential element of social interaction. Although Goffman's conceptualization was intended for face-to-face interactions, Giddens (1984) and Short et al. 
(1976) have taken the concept to mediated interactions. In its early development, the operationalization of copresence was focused on human perception and behavior over an interaction medium. Scholars have examined the human perception of an interaction based on their sense of closeness with another person (Al-Natour, Benbasat, \& Cenfetelli, 2011), shared experiences (Zhu, Benbasat, \& Jiang, 2010), familiarity (Saunders, Rutkowski, Genuchten van, Vogel, \& Orrego, 2011), awareness (Huang, Hong, \& Burtch, 2016), identity (Sia, Tan, \& Wei, 2002), and openness (Hess, Fuller, \& Campbell, 2009).

Research on copresence has evolved along with the ICT's capabilities that can enhance mediated communication. Earlier studies examined a communication medium's capability to facilitate the "perception of being in the same place" and compared the use of mail and telephone as mediums of copresence (Straub, 1994). The focus in these studies has been on the "sense of closeness" felt through the communication medium (O'Leary, Wilson, \& Metiu, 2012). With further advancements in ICTs, the conceptualization of copresence evolved from being a mere perception of closeness to an attribute of technology that creates and enhances the sense of being with the other person (Saunders et al., 2011). This evolution of the construct resulted in the operationalization of copresence as a continuum that ranges from low (low communication cues) to high (virtual world technologies) (Animesh, Pinsonneault, Yang, \& Oh, 2011; Nah, Eschenbrenner, \& DeWester, 2011), where the full level of copresence can only be achieved in face-to-face interaction (Seymour, Riemer, \& Kay, 2018). Therefore, recent literature incorporates social and technical aspects as dimensions of copresence (Davis, Murphy, Owens, Khazanchi, \& Zigurs, 2009).

Although copresence is important for distributed work performance (Altschuller \& Benbunan-Fich, 2010), studies on distributed group performance show that not all activities 
require a high level of copresence (Rice, 1992). For instance, task-oriented activities such as problem-solving tasks require less copresence than human-oriented activities such as consultation and shared decision making (Miranda \& Saunders, 2003). However, studies examining human-machine interactions reveal that humans can react to a machine in ways that are similar to human interaction. Specifically, integrating artificial intelligence technologies (e.g., Amazon's Alexa or Apple's Siri) can convey a sense of realism of the interaction similar in some aspects to that experienced with other human beings (Hess et al., 2009; Seymour et al., 2018), which can induce high levels of copresence.

Other studies have examined the phenomenon beyond technological capabilities and human perception, and instead, focused on the process of communication. Reciprocal exchange of information is shown to have a significant effect on copresence (Miranda \& Saunders, 2003; Ou et al., 2014) because of its effect on decreasing uncertainty (Srivastava \& Chandra, 2018) (Srivastava and Chandra 2018), and increasing the familiarity of participants (Saunders et al., 2011).

\section{The Temporal Dimension of Copresence}

Of particular importance to our study is the effect of time on copresence. Time has been shown to be a critical element for the development of copresence. In particular, studies of virtual worlds have found that interaction duration, frequency, and response time decrease uncertainty and enhance familiarity with the surrounding environment (Animesh et al., 2011; Nah et al., 2011; Saunders et al., 2011), which increases the sense of the presence of others, particularly in conditions of anonymous interactions (Schultze, 2010; Schultze \& Orlikowski, 2010). However, in business-related contexts such as e-commerce (Pavlou, Liang, \& Xue, 2007; Suh \& Lee, 2005) 
and virtual work meetings, those conditions may or may not withhold depending on the associated tasks (Ma \& Agarwal, 2007; Miranda \& Saunders, 2003).

Scholars have studied the effect of duration on perceived presence. For instance, Miranda and Saunders (2003) conducted an experiment that investigated copresence under time constraints. Their results indicated that time is critical for sharing in-depth information that supports copresence in virtual meetings. This is consistent with experiments conducted in health management literature, where the interaction duration affected the process of copresence over time, in which defining an adequate interaction length to each patient position copresence as a timesensitive phenomenon (Finfgeld-Connett, 2006; Zyblock, 2010).

\section{Copresence in Medical Practice}

The concept of copresence is widely used in the medical and nursing literature. That is because the provision of focused care often requires direct interactions between the medical personnel and the patients. Clinical practice requires face to face interactions since both diagnosis and treatment include engagement with patients, touching, and attending to personal needs (Kim et al., 2019). But with the increased use of technology-mediated interactions in medical practice, the need for presence extended beyond the constraints of the immediate space (Finfgeld-Connett, 2006).

Studies have shown that copresence in medical practice depends on the duration of interaction and time spent building psychological closeness that compensates for the absence of physical co-location (Zyblock, 2010). However, in medical emergencies, teams that form for the delivery of healthcare tend to be ad hoc and assembled on a temporary basis, which limits the capacity to build copresence. Since familiarity and interaction durations are nearly absence in 
emergency settings, healthcare literature called for attention to examine copresence in such environments (Barrett, 2017).

\section{Sensemaking Approach to Copresence in Extreme Environments}

At the heart of the care delivery process is the ability to make sense of medical conditions in the face of uncertainty. Patients present with symptoms that may have different interpretations at the same time, which increases the ambiguity of medical cases that fall under the same category. Any communication breakdown between the medical staff can lead to a deterioration of the patient's conditions (Leape \& Berwick, 2005). Therefore, sensemaking is used as a theoretical lens to bind communication and reduce clinical uncertainty (Manojlovich, 2010).

Saunders et al. (2011) argue that actors can perform a set of activities on platforms by using technological tools to increase familiarity and therefore increase the sense of copresence. Specifically, when people are familiar with each other, their cognitive states shape the sensemaking models of the other members who are interacting virtually and help in providing cues during uncertain situations (Srivastava \& Chandra, 2018). Therefore, attention to the processes of sensemaking is critical for understanding copresence.

Sensemaking is "a social process in which organizational members interpret their environment through interactions with others, constructing accounts that allow them to comprehend the world and act collectively" (Maitlis, 2005; Weick, 1995). Weick (1995) suggests that sensemaking is a structured process of reducing ambiguity and uncertainty while making decisions. The process is characterized by seven unique properties, which include the sensemaker's capacity for retrospection, cue extraction, dealing with plausibility, the social and ongoing nature of the sensemaking process, as well as the willingness to create and enact one's identity (the capacity for sensemaking) (Weick, 1988). 
However, in distributed work, these properties of sensemaking are challenged by physical distance. In this context, extracting cues is limited to what the technology enables (Griffith, 1999). For example, if technology capability includes a $360^{\circ}$ view of an environment, the cues extracted will be richer than a limited range camera. Additionally, sensemaking through verbal communication depends greatly on information shared between participants. Sensemaking in distributed work is, therefore, an action-driven process that is guided by collective actions that are mediated through technology.

\section{Research Design and Methods}

To achieve our research objectives, we conducted an ethnographic study since ethnography enables the researcher to become immersed in a culture and directly observe its practices (Maanen, 1979). We followed the semiotic school of ethnography, seeking to develop “thick descriptions" of the research field (Myers, 1999). This paper reports on a total of 16 months of direct engagement with the research site.

\section{Research Site}

Our research site is a group of medical institutions that jointly use a Telestroke system in their Emergency Departments. Telestroke is a telemedicine system that uses a "Hub and Spoke" model in which small hospitals (spokes) are connected to a specialized neuroscience hospital (hub) through a customized system. In Telestroke systems, spokes typically lack neurologists who are specialized in diagnosing and treating acute stroke patients. The hub provides neurologists who can diagnose stroke patients and suggest treatments through the videoconferencing system (Levine \& Gorman, 1999). The system helps save critical time so that 
patients do not have to be transferred to the hub hospital or other medical institutions unless necessary (Wang et al., 2003).

Ischemic stroke is a sudden and unexpected stroke. It is a condition in which reduced blood flow to the brain results in the death of millions of brain cells with every minute that passes. Clinicians have a short "door-to-needle" time which is subjective to time of symptoms, to conduct a complex set of clinical processes involving multiple disciplines, including triage, diagnosis, decision making, and treatment administration, all of which can change with the evolving state of the patient (Hacke et al., 1995). The severity of stroke can be measured by the National Institute Health Stroke Scale (NIHSS) ${ }^{1}$. To objectively quantify the physical impairment caused by a stroke. Treatment often includes the administration of tissue plasminogen activator (tPA), a clot-busting drug that can greatly reduce the probability of developing a disability as a result of the stroke when given within three hours of symptoms onset.

However, the trade-off of this medication is that there is a chance of causing brain bleeding. Therefore, a neurologist must diagnose the type of stroke and assess its severity to decide whether to give rPA and calculate the appropriate dosage. This decision-making process depends on several factors, including the CT image interpretation, NIHSS score to assess the severity, and other patient-related data such as age, weight, and medical history. Other medications may include Aspirin, and patients are transferred to the inpatient ward (Albers et al., 2000).

\footnotetext{
${ }^{1}$. National Institute of Health Stroke Scale (NIHSS) is a tool to aid in stroke assessment. Details are discussed in the appendix.
} 


\section{The Telestroke system}

The Telestroke system comprises different clinical actors who belong to the hub and two spokes. The hub consists of neurologists and neurology registrars (also called fellows in the U.S.) who have to provide the diagnosis based on information provided by actors in the spoke. The actors on the spoke include ER Physicians, Radiologists, Stroke Nurses, ER Nurses, and Radiology Technicians who are present with the patient and are able to collect cues and information about the patient to facilitate prompt and accurate diagnosis. These actors collaborate to enable distant diagnoses and treatments of acute stroke patients who arrive at the spoke hospitals.

In late 2011, the hub initiated the project with one of the spokes and completely implemented it in the two spokes by 2013. The system in the hub is an installed software that is positioned in the neurology staff's office with an additional monitor. The system in the spoke side is located in the Accident and Emergency (A\&E) Department in a room called "Resuscitation Area," the area hosts many beds so that stroke patients can be placed near the Telestroke. The system is installed on a Computer on Wheels (COW), and each spoke has two Telestroke COWs in case of multiple cases arriving at the same time. The videoconferencing capability is based on a one-way video communication in which neurologists can see patients but not vice versa.

We chose a Telestroke system for several reasons. First, Telestroke is situated in an emergency department, which is a setting that requires fast and highly reliable responses. Second, since it is a medical environment, copresence becomes a vital requirement in the process of diagnosis and treatment. Third, it connects several different organizations, including generalist hospitals and a specialized neuroscience institute. This presents opportunities for studying 
copresence, considering the unfamiliarity of actors with others in different organizations. Additionally, it allows us to study the role of technology in facilitating copresence. Finally, in stroke treatments, the short door-to-needle time puts the actors under significant time pressure. For all these reasons, the site was considered an exemplar for our research question.

\section{Data Collection}

We leveraged multiple data sources to enhance the richness and the interpretability of our findings (Venkatesh, Brown, \& Bala, 2013). These data sources include direct observations, shadowing, archival data (e.g. news articles), and semi-structured interviews in the three institutions.

Observations and Shadowing: At the beginning of the study, the first author conducted observations in the hub, i.e., where the neurologists worked. This enabled us to gain an understanding of the practices of Neurologists in the hub during their use of Telestroke. The first author started observations in one of the spoke hospitals and later shadowed stroke nurses throughout the entire Telestroke workflow. Whenever there is a possible stroke case, the first author would be informed to head to the center to shadow the case. An average number of 30 cases per month were conducted using Telestroke. In total, we observed 32 complete stroke cases.

While conducting the observations, the first author did not participate in any of the stroke treatment activities; rather, she observed, took notes, and reflected on the situated actions and interactions of the actors. When she observed events that were unusual or surprising, she spoke to the stroke nurses after the case is finished to ask their interpretations of those events. 
Semi-Structured Interviews: Interviews took place in the three hospitals after completing observations in the hub, and the first spoke. This enabled us to build our interviews on the understanding we had gained of the workflow in both sides of the Telestroke system, and on the theoretical ideas we had concurrently developed. First, we established an initial interview protocol that was based on initial observations. The questions were centered on the following topics: clinical workflow and organizational routines, collaboration within and across sites, interruptions and unexpected events, and organizational changes. We frequently revisited the questions and modified them based on the constructs emerging as we compare observations and interviews. Consent forms were signed before each interview to allow for an audio recording of the conversation. The records were later transcribed and prepared for analysis. By the end of the study, we completed 30 interviews across the three emergency departments.

A few key informants, which include Stroke Nurses, ER Physicians, and Neurologists, were interviewed. Snowball sampling was conducted after interviews with key informants. These medical informants were sometimes difficult to interview due to time availability; they often had medical emergencies they had to attend to, and thus time with them might have to be cut short. The sampling of patients for interviews was also challenging. We had to identify patients who were mentally aware and conscious during the Telestroke process, and most importantly, who could speak coherently. This limited the possible sample of patients, and thus, we were only able to interview three patients.

Meetings: We gained access to four of the quarterly meetings between the three institutions. In each meeting, participants include senior position holders of nurses and physicians from each institution alongside emergency department managers. The content of the meetings includes discussing issues each institution faced from the perspective of different 
actors, solutions are discussed, and decisions are made to be implemented by relevant individuals. We have notes for each meeting.

Archival Data: We collected relevant reports and manuals to track changes in practices and routines systematically. These documents include working routing routines, treatment protocols, and targeted time for each process. We also have 25 news articles that impacted established routines, such as cyber-attacks, privacy concerns, and internet cuts on the national healthcare systems, that provided a broader perspective from the environment surrounding Telestroke.

\section{Data Analysis}

We took an interpretive approach to the analysis of our data (Maanen, 1979). Our analysis was done in two phases: In the first phase, we identified the processes of Telestroke and interactions between the actors across sites. In the second phase, our analysis followed an iterative process between the data and literature to identify key constructs that reflect the variation of copresence over time.

Phase 1: Since we adopt a stream of research that identifies copresence as a capacity for actions (Schultze, 2010; Schultze \& Orlikowski, 2010), we sought to identify the phases of Telestroke processes and practices enacted by actors from the hub and the spokes. To do so, we analyzed the observation notes and interviews to identify the processes enacted in each site.

Phase 2: We followed (Corbin \& Strauss, 1990)'s grounded theory approach to identify emerging themes (Table 1) inductively. By iterating between the data and literature, and then matching the codes with activities found in the first phase of analysis (Saldaña, 2015). This helped us identify variations in copresence in each phase. 
Insert Table 1 about here

\section{Findings}

Our analysis reveals variations in the interactions between the hub and the spokes, which have different copresence requirements. In the first section, we identify the phases and interactions on Telestroke between the hub and spokes.

\section{Telestroke Processes and Interactions}

We identified four main phases that make up the stroke diagnosis to treatment workflow and how Telestroke operates (Figure 1). Briefly, the process involves an initial assessment, interpretation of medical data, teleconsultation, and informed decision. During the initial assessment, the ER team in the spoke (who is with the patient with potential stroke) will perform a CT scan on the patient. The neurologists at the hub will receive the notification to standby and operate Telestroke on their side. At this point, neurologists obtain basic information about the patient on the phone from the ER Physician about the presented symptoms. The actors involved in the interaction in this phase on Telestroke are a neurologist and an ER physician.

Insert Figure 1 about here 
The second phase involves the transmission of CT images to Telestroke by the radiographers at the spokes. Once neurologists get the brain images, they can begin interpreting the given information to diagnose whether there is a stroke or not. Due to clinical complexities, the images may not be clear. They may require the co-interpretation by the radiologists, neurologists, and ER physicians.

If the result of the interpretation phase indicates bleeding in the patient brain, the patient may need to be transferred to another facility for a surgery. However, if the brain image does not reveal bleeding, the neurologist initiates the Telestroke teleconsultation. In this phase, the interaction between the hub and spoke intensifies as neurologists seek more information to diagnose the type of stroke the patient experiencing. Hence, extraction of physical cues is needed to reduce clinical uncertainty.

Stroke assessment is done through NIHSS, which is a tool that objectively quantifies muscle impairment caused by strokes (an example is shown in the appendix). Neurologists at the hub extract physical cues through video by assessing patients' arms, legs, and facial movements and speech abilities. This is done with stroke nurses' assistance at the spoke, who act as a proxy to assist and convey relevant assessment. The interaction in this phase involves neurologists, ER physicians, patients, stroke nurses, and occasionally senior neurologists.

Determining the type and severity of stroke is critical for deciding what treatment and dosage to administer. As described in the methods section, medication includes TPA, which is a blood clot-busting medicine with an associated risk of brain bleeding. Thus, if deemed appropriate, the patient or their family's consent is needed prior to TPA administration. 
Therefore, in the final process of Telestroke, neurologists at the hub communicate their decision of medication to ER physicians and relevant dosage to be given. Depending on stroke severity, the clinicians may continue using Telestroke to follow up with the medication administration process to modify the dosage if needed. Interactions in this phase involve neurologists and ER physicians.

\section{Variations of Copresence}

Results reveal the variation in the levels of copresence needed across the phases of care in Telestroke (Table 2). In the initial assessment phase, interactions between the hub and spokes include verbal communication of patient's information, such as age, time of ED arrival, and time of stroke symptoms (time of onset). Our data indicates minimum copresence needed for such activities. Therefore, we categorize this phase as low-intensity copresence.

Insert Table 2 about here

In the second phase, a CT scan of the patient's brain is taken at the spoke. However, the brain images can be ambiguous and require collaborations between the radiologist and the neurologist. Observations at the hub revealed that there were interactions that occurred outside of Telestroke's system to make up for the absence of physical presence. Neurologists leveraged multiple channels to acquire more clinical information, such as the medical history of previous strokes, to reduce clinical uncertainty. For instance, neurologists use their personal phones to text or call radiologists to consult them on the CT scan results. Another example of such actions is highlighted in Figure 2. 
Insert Figure 1 about here

The observation notes point to the criticality of copresence when ambiguity increases, and situations require a shared understanding. Specifically, the lack of physical presence between the patient and neurologists calls them to acquire information that reduces the distance barrier. However, in-depth interviews with neurologists reveal that copresence is not necessary for such activities. For instance, a neurologist said when asked about the importance of being there at this stage: "From my point of view, it is okay it doesn't affect my work" (Neurologist 4). Therefore, we categorize this phase as conditional copresence, where the criticality of copresence depends on the associated clinical ambiguity.

In the Teleconsultation phase, interactions become complex and involve more actors. Specifically, neurologists have to interact with ER physicians, Stroke Nurses, and patients (if they are conscious enough to communicate). The aim of these interactions is to reduce the uncertainty associated with clinical symptoms within a limited time window (15 minutes). As discussed in the previous section, the teleconsultation phase involves video conferencing between the hub and spoke. However, because of technical constraints, the video only enables the hub to see the spoke (i.e. the patient) but not vice versa.

Our findings reveal the criticality of copresence in the Teleconsultation phase. Specifically, activities that require copresence are the ones that include patients. Whether direct or indirect interactions, neurologists explain that the criticality of copresence is essential regardless of the ambiguity of a stroke. For instance, one physician explained: 
"The interaction is still going to be the same but slightly different if you get what I mean. There's a less human touch to it. So, the gut feeling like how, when you see a patient, "this doesn't feel right," versus over the phone, "I think this doesn't feel right." .... In my experience, when you see this thing, something just doesn't fit into the picture. So, if I have a neurologist who can see the patient personally, it'll be a bit better. ...Seeing the whole person versus what they actually see, only part of the person at a time. Overall, would I be that worried about affecting the diagnosis or the impression? Probably not, but nonetheless, every small bit counts". - ER Physician (7)

During this phase, neurologists have to make the decision whether to give the medication, and it is time-critical. There are severe risks and benefits. Specifically, giving TPA can dissolve the brain blood clot caused by a stroke, but at the same time may cause bleeding in the brain. This increases the pressure of accurate stroke assessment during a teleconsultation phase, which makes it critical for neurologists to feel copresent with the patient. For instance, a neurologist explained:

"So, clinical examination often is a very subtle thing. A flicker of the eye, a little bit of incoordination between both eyes, a tremor of the finger can be very informative. And these are not things that can actually come through a very low-resolution video feed unless, of course, they are reported by a very astute physician on the other side. So as technology develops, who knows, maybe one day we will have augmented reality or virtual reality. All the patients could be projected on the hologram, and all of these things would help us very much. - Neurologist (1)

Moreover, neurologists rely heavily on the spoke team act as a proxy to convey information from their perspective. Thus, their physical presence with the patient is essential to 
the interaction conducted on Telestroke. We, therefore, categorize this phase as a high-intensity copresence.

In the final phase, a decision regarding giving TPA or not is verified by the hub and communicated to the spoke, which has the final decision of administering the medication. Our data reveals that copresence is required only in a few cases where the patients or their families are not convinced with the assessment. Regarding the workflow and interaction between the hub and spokes, we have found no evidence that supports the need for copresence. However, few neurologists pointed out to the need for clinical follow up with patients whose symptoms are complicated and ambiguous. Therefore, we categorize this phase as conditional copresence, where the criticality of copresence depends on the associated clinical ambiguity.

\section{Enactment of Copresence across Time}

Our findings reveal four mechanisms that shaped the variation of copresence across the different phases of the Telestroke process: extracting cues, retrospection, perspective-taking, and selective attention.

Extracting Cues: To effectively conduct medical diagnoses, neurologists need to extract cues of patient symptoms in ways that help them reduce clinical uncertainty. In the absence of physical presence, neurologists extract visual and verbal cues through the Telestroke system's

features. For example, neurologists can control over which part of the patient they want to observe by using a function of zooming in and out. However, they still need to make sense of these observed pieces and come up with a matching assessment. For instance, a neurologist discussed: 
"Not having a direct view of the patient means that you may miss other types of stroke symptoms. So, the NIHSS [assessment scale] is very good for detecting strokes that are involving the MCA [Middle Cerebral Artery Stroke], which means the front part of the brain. But other things are a little bit more subtle [to detect]. For example, just changes in the size of the pupils, or changes with the way they speak. The way they speak is somewhat covered in the NIHSS. But it's only one point there. - Neurologist (11)

Moreover, neurologists on the hub rely heavily on the spoke team to extract sensible cues, especially since they cannot physically examine the patients themselves given physical distance from the patient. The spoke team thus must serve as a surrogate for the hub's neurologists in extracting cues from the patient. For instance, the nurse can speak to the patient and report back to the neurologist, and so on. Any change in patient condition during the teleconsultation will be reported back by nurses (indirect approach to copresence). For instance, a stroke nurse elaborated:

"[The neurologists] are quite dependent on us because they are not physically here to see the patient, so during or after the consultation, if there's anything with the patient's condition we call back, we update them. "I see this improving on the patient's condition, so do you want to make any decision? Do you still want to approve [the medication] or not?" So, they will discuss with the consultant again”. - - Spoke B, Stroke Nurse (2)

Retrospection: Reflecting on experience is one of the salient elements of copresence that emerged from the data. Since stroke cases are not very regular, the spoke team revealed the importance of establishing knowledge and learning from past events to make sense of current cases. This theme was salient in the spoke due to the knowledge distance between the ER team and neurologists who are specialists at assessing strokes. For instance, a neurologist expressed 
her concerns over not being physically present with the patient combined with lack of experience of ER doctors of some stroke types:

"The most difficult part is that we can't really examine a patient, like the routine examination. It can be done by the ER physician. But sometimes there are more ambiguous symptoms associated with strokes. For example, amnesia can be caused by a stroke, and they [ER Physicians] might not be familiar with”. $\quad$ - Neurologist (6)

Therefore, the spokes' medical team can reflect on their experience with Telestroke, even when their role does not include specific activities. This approach bridges the physical and knowledge distance that is created by Telestroke to allow the spoke's clinicians to become better surrogates for the hub's neurologists and achieves a better level of copresence. For instance, an ER physician explained:

"The video bridges "over the phone" conversation. Actually, it enhances verbal conversation. For example, when the patient experiences a facial drop [impaired facial muscles causes the chin to drop]. I communicate verbally to the neurologist and say: "Okay. I think there's a facial droop." Then, the neurologist can zoom in and say, "Okay, yes, I also concur that there is a facial droop." But when it is only verbal communication, I tell him I see a facial drop, and then he asks how much drop, but I actually do not know how much. So, when I saw how he does it with the camera, it is easier later if it happened again. - ER Physician (7)

Perspective Taking: Because of the absence of physical presence in Telestroke interactions, individuals resort to perspective taking to address the challenge of physical separation and enhance copresence. Perspective taking is the process of perceiving others' points 
of view through information acquisition. It depends on perspective making, which is the process through which one explains and conveys their understanding of a certain situation through narration, language, or actions (Boland Jr \& Tenkasi, 1995).

At the hub side, neurologists have limited information about the situation in the ER. Thus, they rely on verbal information and limited view of the patient from the video conferencing channel, and their own experience of regular stroke treatment they administer at their institute. These approaches feed their perspective-taking and therefore assists in making decisions.

At the spoke, the medical team has to convey the situation to neurologists in the hub. In the case of increased ambiguity, the medical team build on assumptions and build a perspective that fits the given situation. For instance, one stroke nurse mentioned how ambiguity is created by the time synchronization issue, where the time at the spoke side of the system was different than the time at the hub side. This difference (although in a few minutes) have created an issue regarding the time of medication decision, which therefore created confusion for Stroke Nurses and used perspective making to assume the situation at the hub. For instance, a nurse explained:

"I did not understand what the neurologist told me on the phone, and it does not match the situation, and I thought he did not know what is going on because he is not here, then I saw that the software timing and the computer timing its different, so initially it was adjusted, but there's one period later the system time was faster, so if approved for rtPA, they click on the system, but it shows 3 minutes earlier than what it already doctor gave, so I assumed the doctor follow the system time.

- Spoke A, Nurse (2) 
Using Telestroke for medical consultation created a virtual space for the medical team to continue their practice. This space allows neurologists in particular to make perspectives of the situation on the other side, which is critical for achieving copresence. For instance, one neurologist consultant shared:

"Because when you have it on the computer or over the screen, there's always this false window between the patient and you. Basically, you don't see the patient in front of you, for example, if I'm talking to you now, or on Skype, this is just different. There's less human touch, and the limitations are also space or where the patient is, what we perceived that they might not be able to show the full body but rather maybe see just the upper body at one time”. - Neurologist (5)

Selective Attention: Time constraints in the process limit actors' ability to enact copresence. Neurologists on the hub pursue this approach to copresence to selectively be attentive to "what matters the most." Specifically, selective attention by focusing on relevant stimuli and ignoring others is a common approach to achieving copresence. In this approach, neurologists choose what is necessary to focus on and therefore be attentive to, and what is not relevant. This is subjective to the examination procedures they want to perform by using functions such as video conferencing, and zooming-in and out. For instance, a neurologist explained how technology-enabled selective attention toward one body part at a time instead of a holistic view of the patient:

"Because right now, the aim of Telestroke is to give us as much situational awareness as can be achieved with current technology. Current technology is very limited in giving us that acuity of information". - Neurologist (1) 
Therefore, technology limitations to capture full presence (similar to complete physical presence) induce selective attention to emerge as an approach for mediated copresence. In contrast to the hub, the spoke team showed little evidence of the use of selective attention as an approach to achieving copresence. We attribute the reason to lack of information flow enabled by technology

\section{Discussion and Contribution}

Past studies have examined copresence as a static performance that is either attained or not through a given communication medium. Our findings from the Telestroke case call for attention to the variation of copresence across different phases of a process. Some phases require a higher intensity of copresence than others. In particular, changes in the level of ambiguity in medical processes result in changes in the required degree of copresence. This induces actors to perform actions that enable them to shift their level of copresence throughout a medical intervention.

This paper identifies four mechanisms that shape the shifts in the degree of copresence: (1) extracting cues, (2) retrospection, (3) perspective-taking, and (4) selective attention. Together, these mechanisms provide a practice perspective that explains how actors vary their level of copresence under conditions of time pressure, where the duration of interactions cannot be extended to reach higher levels of copresence.

The study makes several theoretical and practical contributions. First, studies on copresence highlighted temporal concepts (e.g. immediacy and duration of interactions) as critical for understanding copresence (Harrison, 2018). However, the literature lacks theoretical conceptualization of how copresence varies over time. This study provides a first step in 
developing a theoretical understanding of these variations, particularly in conditions where time pressure and uncertainty are inherent to the working environment. In addition, the findings of the study highlight the need for considering variations in the need for copresence when designing routines or technologies for distant interactions. Attention to the four mechanisms of variation can help the designers of such routines and technologies in enabling shifts in the enactment copresence. Since attaining copresence can be demanding for users of distant interaction systems, the ability to change the level of copresence can help them focus on the more critical parts of their interaction processes. 


\section{References}

Albers, G. W., Bates, V. E., Clark, W. M., Bell, R., Verro, P., et al. 2000. Intravenous tissue-type plasminogen activator for treatment of acute stroke: The Standard Treatment with Alteplase to Reverse Stroke (STARS) study. JAMA, 283(9): 1145-1150.

Al-Natour, S., Benbasat, I., \& Cenfetelli, R. 2011. The adoption of online shopping assistants: Perceived similarity as an antecedent to evaluative beliefs. Journal of the Association for Information Systems, 12(5): 347.

Altschuller, S., \& Benbunan-Fich, R. 2010. Trust, performance, and the communication process in ad hoc decision-making virtual teams. Journal of Computer-Mediated Communication, 16(1): 27-47.

Animesh, A., Pinsonneault, A., Yang, S.-B., \& Oh, W. 2011. An odyssey into virtual worlds: Exploring the impacts of technological and spatial environments on intention to purchase virtual products. MIS Quarterly, 789-810.

Barrett, D. 2017. Rethinking presence: A grounded theory of nurses and teleconsultation. Journal of Clinical Nursing, 26(19-20): 3088-3098.

Boland Jr, R. J., \& Tenkasi, R. V. 1995. Perspective making and perspective taking in communities of knowing. Organization Science, 6(4): 350-372.

Corbin, J. M., \& Strauss, A. 1990. Grounded theory research: Procedures, canons, and evaluative criteria. Qualitative Sociology, 13(1): 3-21.

Davis, A., Murphy, J. D., Owens, D., Khazanchi, D., \& Zigurs, I. 2009. Avatars, people, and virtual worlds: Foundations for research in metaverses. Journal of the Association for Information Systems, 10(2): 90.

Finfgeld-Connett, D. 2006. Meta-synthesis of presence in nursing. Journal of Advanced Nursing, 55(6): 708-714. 
Giddens, A. 1984. The constitution of society: Outline of the theory of structuration. Univ of California Press.

Goffman, E. 1982. Interaction Ritual: Essays in Face to Face Behavior. AldineTransaction.

Griffith, T. L. 1999. Technology features as triggers for sensemaking. Academy of Management Review, 24(3): 472-488.

Hacke, W., Kaste, M., Fieschi, C., Toni, D., Lesaffre, E., et al. 1995. Intravenous thrombolysis with recombinant tissue plasminogen activator for acute hemispheric stroke: The European Cooperative Acute Stroke Study (ECASS). JAMA, 274(13): 1017-1025.

Harrison, A. 2018. The effects of media capabilities on the rationalization of online consumer fraud. Journal of the Association for Information Systems, 19(5): 1.

Henderson, A. 2006. The evolving relationship of technology and nursing practice: Negotiating the provision of care in a high tech environment. Contemporary Nurse, 22(1): 59-65.

Hess, T. J., Fuller, M., \& Campbell, D. E. 2009. Designing interfaces with social presence: Using vividness and extraversion to create social recommendation agents. Journal of the Association for Information Systems, 10(12): 1.

Huang, N., Hong, Y., \& Burtch, G. 2016. Social network integration and user content generation: Evidence from natural experiments. MIS Quarterly, 17-001.

Kim, S. C., Shaw, B. R., Shah, D. V., Hawkins, R. P., Pingree, S., et al. 2019. Interactivity, Presence, and Targeted Patient Care: Mapping e-Health Intervention Effects Over Time for Cancer Patients with Depression. Health Communication, 34(2): 162-171.

Leape, L. L., \& Berwick, D. M. 2005. Five years after To Err Is Human: What have we learned? Jama, 293(19): 2384-2390. 
Levine, S. R., \& Gorman, M. 1999. "Telestroke” The Application of Telemedicine for Stroke. Stroke, 30(2): 464-469.

Liu, N., Kim, J., Jung, Y., Arisy, A., Nicdao, M. A., et al. 2017. Remote monitoring systems for chronic patients on home hemodialysis: Field test of a copresence-enhanced design. JMIR Human Factors, 4(3): e21.

Ma, M., \& Agarwal, R. 2007. Through a glass darkly: Information technology design, identity verification, and knowledge contribution in online communities. Information Systems Research, 18(1): 42-67.

Maanen, J. V. 1979. The Fact of Fiction in Organizational Ethnography. Administrative Science Quarterly, 24(4): 13.

Maitlis, S. 2005. The social processes of organizational sensemaking. Academy of Management Journal, 48(1): 21-49.

Manojlovich, M. 2010. Nurse/physician communication through a sensemaking lens: Shifting the paradigm to improve patient safety. Medical Care, 48(11): 941-946.

Miranda, S. M., \& Saunders, C. S. 2003. The social construction of meaning: An alternative perspective on information sharing. Information Systems Research, 14(1): 87-106.

Myers, M. D. 1999. Investigating information systems with ethnographic research.

Communications of the Association for Information Systems, 2(1): 23.

Nah, F. F.-H., Eschenbrenner, B., \& DeWester, D. 2011. Enhancing brand equity through flow and telepresence: A comparison of 2D and 3D virtual worlds. MIS Quarterly, 731-747.

O'Leary, M. B., Wilson, J. M., \& Metiu, A. 2012. Beyond being there: The symbolic role of communication and identification in the emergence of perceived proximity in geographically dispersed work. 
Ou, C. X., Pavlou, P. A., \& Davison, R. 2014. Swift guanxi in online marketplaces: The role of computer-mediated communication technologies. MIS Quarterly, 38(1): 209-230.

Paul, C. L., Cox, M. E., Small, H. J., Boyes, A. W., O’Brien, L., et al. 2017. Techniques for improving communication of emotional content in text-only web-based therapeutic communications: Systematic review. JMIR Mental Health, 4(4): e46.

Pavlou, P. A., Liang, H., \& Xue, Y. 2007. Understanding and mitigating uncertainty in online exchange relationships: A principal-agent perspective. MIS Quarterly, 105-136.

Rice, R. E. 1992. Task analyzability, use of new media, and effectiveness: A multi-site exploration of media richness. Organization Science, 3(4): 475-500.

Saldaña, J. 2015. The coding manual for qualitative researchers. Sage.

Saunders, C., Rutkowski, A. F., Genuchten van, M., Vogel, D., \& Orrego, J. M. 2011. Virtual space and place: Theory and test. MIS Quarterly, 1079-1098.

Schultze, U. 2010. Embodiment and presence in virtual worlds: A review. Journal of Information Technology, 25(4): 434-449.

Schultze, U., \& Orlikowski, W. J. 2010. Research commentary-Virtual worlds: A performative perspective on globally distributed, immersive work. Information Systems Research, 21(4): 810-821.

Seymour, M., Riemer, K., \& Kay, J. 2018. Actors, avatars and agents: Potentials and implications of natural face technology for the creation of realistic visual presence.

Journal of the Association for Information Systems, 19(10): 953-981.

Short, J., Williams, E., \& Christie, B. 1976. The Social Psychology of Telecommunications. London: Wiley. 
Sia, C.-L., Tan, B. C., \& Wei, K.-K. 2002. Group polarization and computer-mediated communication: Effects of communication cues, social presence, and anonymity. Information Systems Research, 13(1): 70-90.

Srivastava, S. C., \& Chandra, S. 2018. Social presence in virtual world collaboration: An uncertainty reduction perspective using a mixed methods approach. MIS Quarterly, 42(3): 779-803.

Straub, D. W. 1994. The Effect of Culture on IT Diffusion: E-Mail and FAX in Japan and the US. Information Systems Research, 5(1): 23-47.

Suh, K.-S., \& Lee, Y. E. 2005. The effects of virtual reality on consumer learning: An empirical investigation. MIS Quarterly, 673-697.

Venkatesh, V., Brown, S. A., \& Bala, H. 2013. Bridging the qualitative-quantitative divide: Guidelines for conducting mixed methods research in information systems. MIS Quarterly, 21-54.

Wang, S., Lee, S. B., Pardue, C., Ramsingh, D., Waller, J., et al. 2003. Remote evaluation of acute ischemic stroke: Reliability of National Institutes of Health Stroke Scale via telestroke. Stroke, 34(10): e188-e191.

Weick, K. E. 1988. Enacted sensemaking in crisis situations [1]. Journal of Management Studies, 25(4): 305-317.

Weick, K. E. 1995. Sensemaking in organizations, vol. 3. Sage.

Yoo, Y., \& Alavi, M. 2001. Media and group cohesion: Relative influences on social presence, task participation, and group consensus. MIS Quarterly, 371-390.

Zhu, L., Benbasat, I., \& Jiang, Z. 2010. Let's shop online together: An empirical investigation of collaborative online shopping support. Information Systems Research, 21(4): 872-891. 
Zyblock, D. M. 2010. Nursing presence in contemporary nursing practice. Nursing Forum, 45 : 120-124. Wiley Online Library. 


\title{
Appendix: Sample of the National Institute Health Stroke Scale (NIHSS)
}

\author{
National Institute Health Stroke Scale (NIHSS) is a tool to aid in stroke assessment.
}

Details are discussed in the appendix. including facial, limbs, sensory, and language disabilities

caused by acute strokes. The tool provides a high degree of inter-observer agreement to increase

reliability (Goldstein et al. 1989).

\begin{tabular}{|c|c|c|c|c|}
\hline \multicolumn{2}{|c|}{ National Institutes of Health Stroke Scale(NIHSS) } & \multicolumn{3}{|l|}{ Score } \\
\hline Instructions & Scale Definition & Baseline & $\begin{array}{l}24 \text { Hrs } \\
\text { Post TPA }\end{array}$ & Discharge \\
\hline \multicolumn{5}{|l|}{ Date/Time } \\
\hline 1a. LOC & $\begin{array}{l}0=\text { Alert keenly responsive } \\
1=\text { Not Alert but arousable by minor stimulation to obey, answer, respond } \\
2=\text { Not Alert; requires repeat stimulation, obtunded, requires strong stimuli } \\
3=\text { Reflex motor or autonomic effects response, totally unresponsive, flaccid }\end{array}$ & & & \\
\hline $\begin{array}{l}\text { 1b. LOC Questions. Ask the } \\
\text { patient the month \& age }\end{array}$ & $\begin{array}{l}0=\text { Answers both questions correctly } \\
1=\text { Answers one question correctly } \\
2=\text { Answers neither questions correctly }\end{array}$ & & & \\
\hline $\begin{array}{l}\text { 1c. LOC Commands. Ask to open } \\
\& \text { close eyes, then grip \& release } \\
\text { with non-paretic hand. }\end{array}$ & $\begin{array}{l}0=\text { Performs both tasks correctly } \\
1=\text { Performs one task correctly } \\
2=\text { Performs neither task correctly }\end{array}$ & & & \\
\hline $\begin{array}{l}\text { 2. Best Gaze. Asked to follow with } \\
\text { eyes thru horizontal plane (or } \\
\text { oculocephalic maneuver). }\end{array}$ & $\begin{array}{l}0=\text { Normal } \\
1=\text { Partial Gaze Palsy; gaze is abnormal in } 1 \text { or both eyes, but forced deviation } \\
\text { or total gaze paresis is not present. } \\
2=\text { Forced deviation; total gaze paresis not overcome by oculocephalic man. }\end{array}$ & & & \\
\hline $\begin{array}{l}\text { 3. Visual fields (quadrants) tested } \\
\text { with finger counting or visual } \\
\text { threat.(done by confrontation) }\end{array}$ & $\begin{array}{l}0=\text { No visual loss } \\
1=\text { Partial hemianopia } \\
2=\text { Complete hemianopia } \\
3=\text { Bilateral hemianopia (including cortical blindness) }\end{array}$ & & & \\
\hline $\begin{array}{l}\text { 4. Facial Palsy. Asked to show } \\
\text { teeth \& raise eyebrows }\end{array}$ & $\begin{array}{l}0=\text { Normal symmetrical movement } \\
1=\text { Minor paralysis (flattened nasolabial fold, asymmetry on smiling) } \\
2=\text { Partial paralysis (total or near total paralysis of lower face) } \\
3=\text { Complete paralysis of one or both sides (no upper/lower face mvmt) }\end{array}$ & & & \\
\hline $\begin{array}{l}\text { 5. Motor Arm. Asked to extend } \\
\text { arms (palm down) } 90^{\circ} \text { (if sitting) or } \\
45^{\circ} \text { (if supine) \& hold for } 10 \\
\text { seconds. Begin with non-paretic } \\
\text { limb. }\end{array}$ & $\begin{array}{l}0=\text { No drift; limb holds } 90^{\circ}\left(\text { or } 45^{\circ}\right) \text { for full } 10 \text { seconds } \\
1=\text { Drift, limb holds } 90^{\circ}\left(\text { or } 45^{\circ}\right) \text { but drifts down before full } 10 \text { seconds but does } \\
\text { not hit bed or other support } \\
2=\text { Some effort against gravity, limb cannot get to or maintain (if cued } 90^{\circ} \text { or } \\
\left.45^{\circ}\right) \text { drifts down to bed, but has some effort against gravity. } \\
3=\text { No effort against gravity, limb falls } \\
4=\text { No movement } \\
* \mathrm{UN}=\text { Amputation, joint fusion: Explain }\end{array}$ & $\begin{array}{l}\text { Left: } \\
\text { Right: }\end{array}$ & $\begin{array}{l}\text { Left: } \\
\text { Right: }\end{array}$ & $\begin{array}{l}\text { Left: } \\
\text { Right: }\end{array}$ \\
\hline $\begin{array}{l}\text { 6. Motor Leg. While supine, asked } \\
\text { to hold leg at } 30^{\circ} \text { for } 5 \text { seconds. }\end{array}$ & $\begin{array}{l}0=\text { No drift } \text { leg holds } 30^{\circ} \text { for full } 5 \text { seconds } \\
1=\text { Drift }, \text { leg falls but does not hit bed } \\
2=\text { Some effort against gravity, falls to bed w } / \text { in } 5 \mathrm{sec} \\
3=\text { No effort against gravity; leg falls to bed immediately } \\
4=\text { No movement } \\
* U N=\text { Amputation, joint fusion: Explain }\end{array}$ & $\begin{array}{l}\text { Left: } \\
\text { Right: }\end{array}$ & $\begin{array}{l}\text { Left: } \\
\text { Right: }\end{array}$ & $\begin{array}{l}\text { Left: } \\
\text { Right: }\end{array}$ \\
\hline $\begin{array}{l}\text { 7.Limb Ataxia. Finger - nose \& } \\
\text { heel - shin test on both } \\
\text { sides }\end{array}$ & $\begin{array}{l}0=\text { Absent } \\
1=\text { Present in one limb } \\
2=\text { Present in two limbs } \\
* \mathrm{UN}=\text { Amputation, joint fusion: Explain }\end{array}$ & & & \\
\hline
\end{tabular}




\begin{tabular}{|c|c|c|c|}
\hline $\begin{array}{l}\text { 8. Sensory. Sensation or grimace } \\
\text { to pin prick or withdrawal from } \\
\text { noxious stimuli to limbs in } \\
\text { obtunded or aphasic patient. }\end{array}$ & $\begin{array}{l}0=\text { Normal, no sensory loss } \\
1=\text { Mild/moderate sensory loss; may be dulled/"Not as sharp" } \\
2=\text { Severe/total sensory loss; not aware of face/arm/leg being touched. }\end{array}$ & & \\
\hline $\begin{array}{l}\text { 9.Best Language. Describe what is } \\
\text { happening in picture, name items } \\
\text { of figures, read list of sentences on } \\
\text { attached figures. }\end{array}$ & $\begin{array}{l}0=\text { No aphasia, normal } \\
1=\text { Mild } / \text { moderate aphasia; some loss of fluency / comprehension, without } \\
\text { limitation of expression of ideas. (can identify what is happening in picture) } \\
2=\text { Severe aphasia; (cannot identify pictures) } \\
3 \text { = Mute; global aphasia; no usable speech; or auditory comprehension }\end{array}$ & & \\
\hline $\begin{array}{l}\text { 10.Dysarthria. Read or repeat } \\
\text { words from list. }\end{array}$ & $\begin{array}{l}0=\text { Normal articulation } \\
1=\text { Mild } / \text { Moderate; slurs some words; understood w/some difficulty. } \\
2=\text { Severe, so slurred as to be unintelligible; mute/anarthric } \\
* \mathrm{UN}=\text { Intubated or other physical barrier. Explain }\end{array}$ & & \\
\hline $\begin{array}{l}\text { 11.Extinction \& Inattention. } \\
\text { Look at visual (from \#3) and double } \\
\text { simultaneous tactile. Do both arms } \\
\text { \& legs. }\end{array}$ & $\begin{array}{l}0=\text { No abnormality } \\
1=\text { Visual, tactile, auditory, spatial or personal inattention or extinction to } \\
\text { bilateral stimulation in one sensory modalities. } \\
2=\text { Profound hemi-inattention or inattention to more than one modality; } \\
\text { does not recognize own hand; orients to only one side of space. }\end{array}$ & & \\
\hline \multicolumn{2}{|l|}{$* \mathrm{UN}=$ untestable } & & \\
\hline \multicolumn{2}{|l|}{ Initials } & & \\
\hline \multicolumn{2}{|l|}{ Physician or Nurse Signature } & & \\
\hline
\end{tabular}




\section{List of Tables}

TABLE 1

\section{Thematic Analysis}

\begin{tabular}{|l|l|}
\hline SAMPLE QUOTES & Emerging Themes \\
\hline $\begin{array}{l}\text { "There's a less human touch to it, o the gut feeling like how when } \\
\text { you see a patient, "this doesn't feel right," versus over the phone, } \\
\text { "I think this doesn't feel right." (Neurologist 7) }\end{array}$ & Need for Copresence \\
\hline $\begin{array}{l}\text { "They [patients] know somebody is just looking at them for } \\
\text { behind the camera, and yet they're being not able to ask the person } \\
\text { behind the camera things to reduce the anxiety of what's going } \\
\text { on." (Stroke Nurse 1) }\end{array}$ & Uncertainty \\
\hline $\begin{array}{l}\text { "If you want to ask specific questions to the patient, we can't ask } \\
\text { directly so we can only go to the ED doctors and tell them what to } \\
\text { ask, and they need to ask the patient, and they need to report back } \\
\text { to us." Neurologist 6 }\end{array}$ & Indirect Copresence \\
\hline $\begin{array}{l}\text { A flicker of the eye, a little bit of incoordination between both } \\
\text { eyes, a tremor of the finger can be very informative. (Neurologist } \\
1 \text { ) }\end{array}$ & Extracting Cues \\
\hline $\begin{array}{l}\text { "I can suggest based on my experience, but the patient situation is } \\
\text { not clear to me either." (Observation Note 22, Stroke Nurse) }\end{array}$ & Retrospection \\
\hline $\begin{array}{l}\text { "The aim of Telestroke is to give us as much situational awareness } \\
\text { as can be achieved with current technology." (Neurologist 1) }\end{array}$ & Selective Attention \\
\hline $\begin{array}{l}\text { "Aggregating all the information I get from the radiologist, the } \\
\text { stroke nurse, and the doctor on the ground, I could make sense of } \\
\text { the situation." } \\
\text { (Neurologist 2) }\end{array}$ & Perspective Taking \\
\hline
\end{tabular}


TABLE 2

Variations in Levels of Copresence across Telestroke Phases

\begin{tabular}{|l|l|l|l|}
\hline PHASES & Interaction & $\begin{array}{l}\text { Communication } \\
\text { Medium }\end{array}$ & $\begin{array}{l}\text { Criticality of } \\
\text { Copresence }\end{array}$ \\
\hline Initial Assessment & $\begin{array}{l}\text { Neurologist-ER } \\
\text { Doctors }\end{array}$ & Verbal & Low \\
\hline $\begin{array}{l}\text { Interpretation of } \\
\text { Clinical Data }\end{array}$ & $\begin{array}{l}\text { Neurologist-ER } \\
\text { Doctors }\end{array}$ & Verbal & Low \\
\cline { 2 - 4 } & $\begin{array}{l}\text { Neurologist- } \\
\text { Radiologists }\end{array}$ & Verbal and Visual & Conditional on Ambiguity \\
\hline Teleconsultation & $\begin{array}{l}\text { Neurologist-ER } \\
\text { Doctors and Stroke } \\
\text { Nurses }\end{array}$ & Verbal & High \\
\cline { 2 - 4 } & $\begin{array}{l}\text { Neurologists - } \\
\text { Patients }\end{array}$ & Verbal and Visual & High \\
\hline Informed Decision & $\begin{array}{l}\text { Neurologists - ER } \\
\text { Doctors }\end{array}$ & Verbal & Conditional on Ambiguity \\
\hline
\end{tabular}




\section{List of Figures}

FIGURE 1

Phases of Telestroke and activities conducted by the actors at the hub and spokes

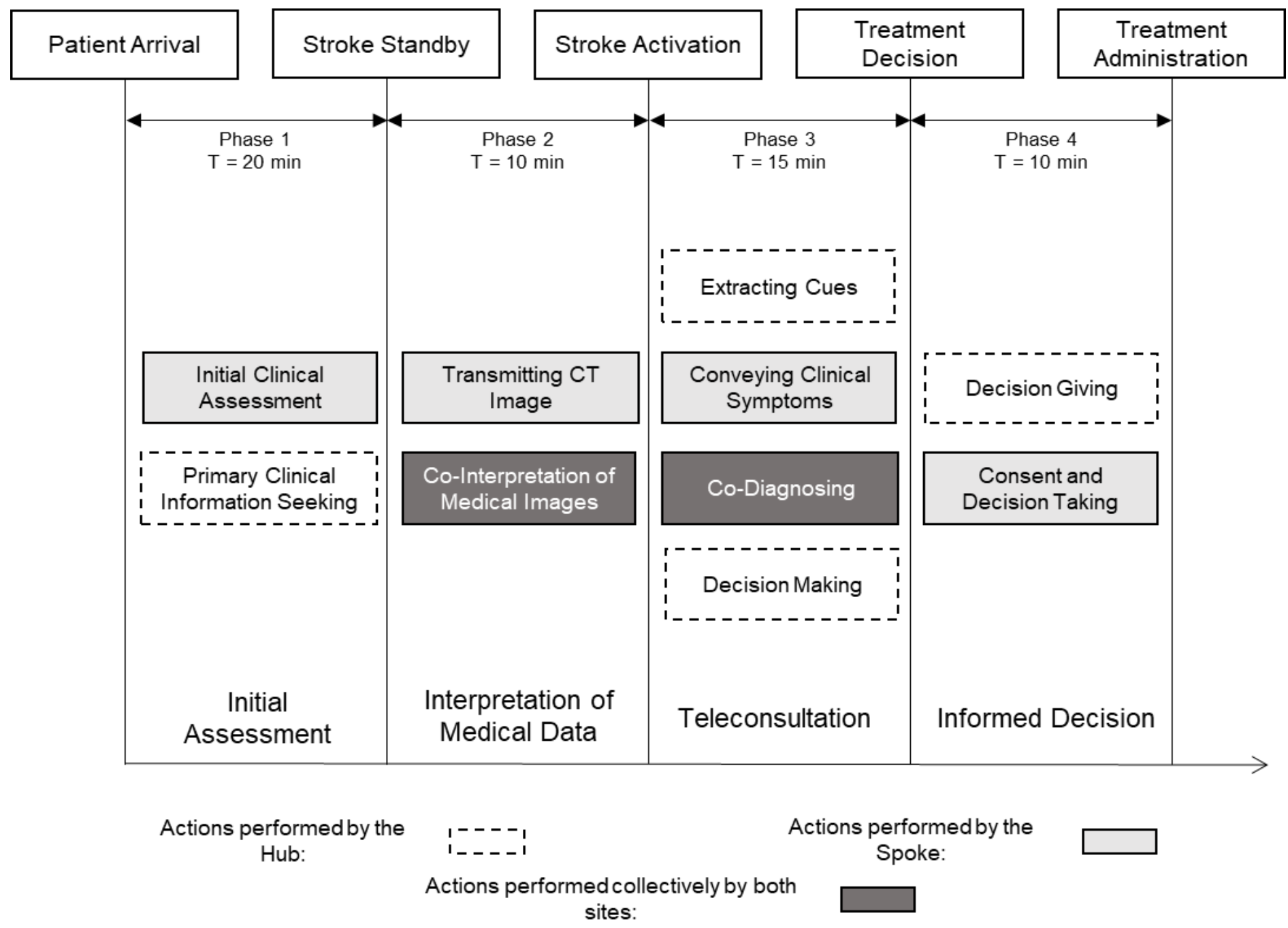




\section{FIGURE 2}

\section{Observation Note \#5 from the Hub}

Site: Hub

Spoke: A

Duration: 40 minutes

1:40 PM: The neurologist is looking at the CT scan but cannot identify the stroke. She picks up the phone and asks the nurse in the spoke to hand the phone to the radiologist. After a while, she asks the radiologist for his opinion on the image. She suspects multiple strokes in the brain.

1:46 PM: Closes the phone with the neurologist and operates some filters on Telestroke to identify the numbers and positions of strokes.

1:49 PM: After some attempts, the neurologist is still skeptical about the CT scan interpretation. She calls a senior neurologist to ask about his opinion of the images. She later asks the ER physician again for initial symptoms that appear on the patient to make sense of the case. In cases of non-Telestroke cases, Neurologists elaborated that they do not need these steps given their physical presence with the patient where they can investigate by themselves. 